\title{
MODELO DE FINLAY Y WILKINSON VS. EL MODELO AMMI PARA ANALIZAR LA INTERACCIÓN GENOTIPO-AMBIENTE EN SORGO
}

\author{
FINLAY AND WILKINSON MODEL VS. AMMI MODEL IN THE ANALYSIS OF \\ GENOTYPE-ENVIRONMENT INTERACTION IN SORGHUM
}

\author{
Héctor Williams Alanís ${ }^{1 *}$, Víctor Pecina Quintero ${ }^{2}$, Francisco Zavala García ${ }^{1}$, Noé Montes García ${ }^{3}$, \\ A. Josué Gámez Vázquez ${ }^{2}$, Gerardo Arcos Cavazos ${ }^{4}$, Miguel A. García Gracia ${ }^{3}$, Salvador Montes \\ Hernández $^{2}$ y Leticia Alcalá Salinas ${ }^{5}$
}

\begin{abstract}
${ }^{1}$ Facultad de Agronomía, Universidad Autónoma de Nuevo León. Carr. Zazua-Marín, km 17.5. 66700, Marín, N.L., México. ${ }^{2}$ Campo Experimental Bajío, Instituto Nacional de Investigaciones Forestales Agrícolas y Pecuarias (INIFAP). Apdo. Postal 112. 38010, Celaya, Gto., México. ${ }^{3}$ Campo Experimental Río Bravo, INIFAP. Apdo. Postal 172. 88900, Río Bravo, Tam., México. ${ }^{4}$ Campo Experimental Sur de Tamaulipas, INIFAP. Carr. Tampico-Cd. Mante km. 55. Apdo. Postal C-1 Suc. Aeropuerto. 89339 Tampico, Tam., México. ${ }^{5}$ Delegación Tamaulipas, Secretaria de Agricultura (SAGARPA). Aluminio y 5 de Mayo. 87600, San Fernando, Tam., México.
\end{abstract}

* Autor para correspondencia (hectorwilliamsa@yahoo.com.mx)

\section{RESUMEN}

Con el objetivo de evaluar el rendimiento de grano y la estabilidad de genotipos de sorgo (Sorghum bicolor L. Moench), se sembraron 44 híbridos en 16 ambientes, durante los años 2001 y 2002 en los Estados de Tamaulipas, Nuevo León y Coahuila, México. La interacción genotipo-ambiente se estimó con el modelo de regresión de Finlay y Wilkinson y con el de efectos principales aditivos e interacciones multiplicativas (AMMI). El modelo AMMI resultó más efectivo para caracterizar el comportamiento de los genotipos que el análisis de Finlay y Wilkinson. Los cuatro primeros componentes principales (ACP) del modelo AMMI fueron significativos (P < 0.01), y explicaron 28, 19, 10 y $9 \%$ de la suma de cuadrados de la interacción. En total, el modelo AMMI retuvo $75 \%$ de la suma de cuadrados total, mientras que el residual sólo representó $4 \%$, lo que sugiere que el modelo AMMI fue efectivo para explicar el comportamiento de los genotipos. En este estudio los híbridos de sorgo más estables fueron 'RB-119x435', 'Magnum', 'RB106x25CEA', 'RB-118x430REA', 'RB-119x430CEA', 'Asgrow Coral' y 'WAC-690' . No se observó asociación entre los híbridos más productivos y los mejores ambientes de producción.

Palabras clave: Sorghum bicolor, híbridos, estabilidad, noreste de México.

\section{SUMMARY}

To assess grain yield and stability of sorghum genotypes of (Sorghum bicolor L. Moench), 44 hybrids were sowed in 16 environments during 2001 and 2002 in the states of Tamaulipas, Nuevo León and Coahuila, México. The genotype-environment interaction was estimated by the Finlay and Wilkinson is regression model and by the additive main effects and multiplicative interaction model (AMMI). The AMMI model was more effective for characterizing the behavior of the studied genotypes, than Finlay and Wilkinson is regression analysis. The first four principal compo- nents (ACP) of the AMMI model were significant $(P<0.01)$ and explained 28, 19, 10 and $9 \%$ of the sum of squares of the interaction. In total, the AMMI model retained $75 \%$ of the total sum squares, while the residual only represented $4 \%$. Thus, the AMMI model effectively explains genotype performance. In this study, the most stable sorghum hybrids were ' $R B-119 x 435$ ', 'Magnum', 'RB-106x25CEA', 'RB-118x430REA', 'RB119x430CEA', 'Asgrow Coral' and 'WAC-690' . No association was observed between the most productive hybrids and the best environments.

Index words: Sorghum bicolor, hybrids, stability of grain yield, northeast México.

\section{INTRODUCCIÓN}

El noreste de México es la región más productora de sorgo (Sorghum bicolor L. Moench) del país, donde se siembran anualmente más de un millón de hectáreas (66 $\%$ de la superficie total). En la región se utilizan híbridos de alto potencial de rendimiento, en riego y temporal (secano), los cuales presentan una respuesta diferencial a las diversas condiciones edafoclimáticas de la región. Es necesario entonces disponer de genotipos con altos rendimientos y estables.

La estabilidad permite al genotipo ajustar su capacidad productiva a la más amplia variación ambiental (Lin et $a l .$, 1986). Al respecto, se han desarrollado procedimientos univariados (Yates y Cochran, 1938; Finlay y Wilkinson, 1963; Eberhart y Russell, 1966) y multivariados para estimar la estabilidad y la interacción genotipoambiente (G x A) (Mandel, 1971; Brennan et al., 1981; 
Crossa et al., 1990). Los métodos multivariados son más adecuados para evaluar la estabilidad porque permiten describir e interpretar los efectos de la interacción G x A (Gauch y Furnas, 1991). El modelo AMMI (additive main effects and multiplicative interactions) es uno de los más empleados para estimar la interacción G x A (Zobel et al., 1988; Crossa et al., 1990; Chisi et al., 1996; Brancourt-Hulmel y Lecomte, 2003), ya que considera que los efectos de los factores principales (genotipos y ambiente) son aditivos y lineales, lo que permite estimarlos a través del análisis de varianza; mientras que la interacción $\mathrm{G}$ x A tiene efectos multiplicativos que pueden ser explicados por el análisis de componentes principales (ACP). Además, el modelo AMMI permite el análisis de la interacción con más de un procedimiento estadístico y disponer de estimados exactos del rendimiento (Gauch, 1992).

En el ACP los valores de los ejes describen los patrones de respuesta de los genotipos, por medio de un índice de sensibilidad. Los valores positivos describen los genotipos con mejor comportamiento en ambientes de alto rendimiento, y lo contrario ocurre con los puntajes negativos. Un valor de cero o próximo a éste corresponde a un genotipo con sensibilidad media. Según Crossa et al. (1990), las puntuaciones AMMI no miden la estabilidad sino el grado de interacción del genotipo con el ambiente. Cuando en el ACP un genotipo presenta un valor próximo a cero, la interacción es pequeña; cuando ambos valores del ACP tienen el mismo signo, su interacción es positiva; si son diferentes es negativa. Hernández y Crossa (2000), indican y ejemplifican la ventaja de la gráfica AMMI Biplot para explicar la interacción genotipo-ambiente. Según Yan et al. (2000) dada la necesidad de evaluar a los genotipos en un limitado número de ambientes, la mejor localidad puede ser la que disponga de altos valores del componente principal uno (CP1) y pequeños valores de componente principal dos (CP2). Crossa et al. (1991) señalan también que localidades con valores CP1 cercanos a cero tienen poca interacción y baja discriminación de genotipos.

El objetivo del presente trabajo fue comparar el modelo de Finlay y Wilkinson vs. el modelo AMMI para analizar la interacción genotipo-ambiente de híbridos de sorgo.

\section{MATERIALES Y MÉTODOS}

Se evaluaron 35 híbridos experimentales generados por el Instituto Nacional de Investigaciones Forestales, Agrícolas y Pecuarias (INIFAP) y nueve híbridos de compañías comerciales (Cuadro 1). Los 44 genotipos se evaluaron en 16 ambientes del noreste de México, que comprende los Estados de Tamaulipas, Nuevo León y
Coahuila, durante los años 2001 y 2002. La localización geográfica de las localidades fueron las siguientes: Río Bravo, Tam. (25 $5^{\circ}$ ' LN/ $98^{\circ}$ 01' LO); Empalme, Matamoros, Tam. (25 54' LN/ $\left.97^{\circ} 47^{\prime} \mathrm{LO}\right)$; El Vaso, Matamoros, Tam. (25 54' LN/ $\left.97^{\circ} 49^{\prime} \mathrm{LO}\right)$; Abasolo,

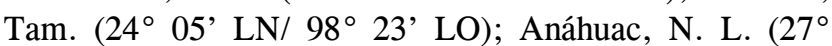
$\left.13^{\prime} \mathrm{LN} / 100^{\circ} 08^{\prime} \mathrm{LO}\right)$; Zaragoza, Coah. $\left(28^{\circ} 35^{\prime} \mathrm{LN} /\right.$ $\left.100^{\circ} 54^{\prime} \mathrm{LO}\right)$; Est. Cuauhtémoc, Tam. $\left(22^{\circ} 33^{\prime} \mathrm{LN} / 98^{\circ}\right.$ 09' LO); y González Villarreal, Tam. $\left(25^{\circ} 23^{\prime} \mathrm{LN} / 97^{\circ}\right.$ $58^{\prime}$ LO). En el Cuadro 2 se presentan las condiciones de siembra en cada ambiente. Los genotipos se distribuyeron en un diseño de bloques completos al azar con cuatro repeticiones. La parcela experimental fue de dos surcos de $5 \mathrm{~m}$ de largo y $0.80 \mathrm{~m}$ de ancho. Se utilizó una densidad de población de 150 mil plantas ha ${ }^{-1}$ en temporal (secano) y 250 mil plantas ha $^{-1}$ en riego. El manejo del cultivo se hizo de acuerdo con las recomendaciones que el INIFAP sugiere para el cultivo de sorgo en la región (Montes y Aguirre, 1992).

La variable analizada fue el rendimiento de grano $(\mathrm{kg}$ ha $^{-1}$ ). Con el programa de cómputo del USDA-ARSUSPSNL se obtuvo el análisis propuesto por Finlay y Wilkinson (1963), que incluye el análisis de regresión conjunto, la regresión de ambientes, de genotipos y el residual. También se aplicó el modelo AMMI que considera el análisis de varianza para efectos de genotipo y ambiente, y un análisis de componentes principales para la interacción genotipo-ambiente (Gollob, 1968; Zóbel, 1990; Crossa et al., 1990).

\section{RESULTADOS Y DISCUSIÓN}

En el análisis de varianza combinado (Cuadro 3) hubo diferencias $(\mathrm{P} \leq 0.01)$ entre ambientes y genotipos, $\mathrm{y}$ efectos de la interacción $\mathrm{G}$ x A, lo que significa que los híbridos respondieron de manera diferencial a la variación ambiental. La suma de cuadrados de ambientes explicó 61 $\%$ de la variación total, lo que confirma una fuerte variación en las condiciones climáticas y edáficas, entre ambientes. El análisis de regresión de Finlay y Wilkinson (1963) (regresión conjunta, regresión debida a los genotipos y la regresión debida a ambientes) no fue el más apropiado para caracterizar los genotipos, ya que la variación debida a estas fuentes sólo explicó $14 \%$ de la varianza debida a la interacción ( $\mathrm{G} \times \mathrm{A}$ ) con $9 \%$ de los grados de libertad (Shukla, 1972). Además, la regresión conjunta resultó no significativa ya que sólo explicó $0.3 \%$ de la varianza de dicha interacción. La fracción residual de la interacción $\mathrm{G} \times \mathrm{A}$ fue significativa $(\mathrm{P} \leq 0.01)$, lo que confirma que el modelo de regresión no es apropiado para describir el comportamiento de los genotipos en estudio, ya que no reduce la varianza y la suma de cuadrados de esta fracción representa $86 \%$ de la interacción. 


\begin{tabular}{llll}
$\begin{array}{l}\text { Cuadro 1. Híbridos experimentales y comerciales de sorgo evaluados en } \\
\text { México, durante los ciclos otoño-invierno }\end{array}$ & \multicolumn{1}{c}{ ambientes del noreste de } \\
\multicolumn{1}{c}{ Híbrido } & Número & \multicolumn{1}{c}{ Híbrido } \\
\hline Número* & 'RB-118x430' & G23 & 'RB-119x435' \\
\hline G1 & 'RB-118x435' & G24 & 'RB-119x216' \\
G2 & 'RB-118x204' & G25 & 'RB-119xBER' \\
G3 & 'RB-118x216' & G26 & 'RB-119x430CEA' \\
G4 & 'RB-118x430REA' & G27 & 'RB-119x25CEA' \\
G5 & 'RB-118x430CEA' & G28 & 'RB-116x430' \\
G6 & 'RB-118x25CEA' & G29 & 'RB-116x435' \\
G7 & 'RB-Patrón' & G30 & 'RB-116x216' \\
G8 & 'RB-104x435' & G31 & 'RB-116x25CEA' \\
G9 & 'RB-104x216' & G32 & 'RB-106x430' \\
G10 & 'RB-104x430CEA' & G33 & 'RB-106x435' \\
G11 & 'RB-104xBER' & G34 & 'RB-106x204' \\
G12 & 'RB-5x435' & G35 & 'RB-106x216' \\
G13 & 'RB-5x204' & G36 & 'RB-106x25CEA' \\
G14 & 'RB-5x216' & G37 & 'Asgrow Coral' \\
G15 & 'RB-5x430REA' & G38 & 'RB-4040' \\
G16 & 'RB-5x430CEA' & G39 & 'RB-3030' \\
G17 & 'RB-5x25CEA' & G40 & 'RB-3006' \\
G18 & 'RB-125x430CEA' & G41 & 'Magnum 380' \\
G19 & 'RB-125x25CEA' & G42 & 'Pioneer 8313' \\
G20 & 'RB-125xBER' & G43 & 'Dekalb D55' \\
G21 & 'RB-119x430' & G44 & 'WAC 690' \\
G22 & & & \\
\hline *1 a 7, 9 a 36: híbridos & experimentales de INIFAP, Campo Experimental Río Bravo; 8 y 38 a 44: híbridos \\
comerciales. & &
\end{tabular}

Cuadro 2. Condiciones de siembra en los ambientes evaluados en el noreste de México.

\begin{tabular}{lllllc}
\hline Núm. & Localidades 2001 & Cond. & Núm & Localidades 2002 & Cond. \\
\hline A1 & Río Bravo, Tam. & T & A9 & Río Bravo, Tam. & T \\
A2 & Empalme, Tam. & T & A10 & Empalme, Tam. & T \\
A3 & El Vaso, Tam. & T & A11 & El Vaso, Tam. & T \\
A4 & Abasolo, Tam. & R & A12 & Abasolo, Tam. & P \\
A5 & Río Bravo, Tam. & R & A13 & Río Bravo, Tam. & R \\
A6 & Anahuac, N. L. & R & A14 & González V, Tam. & T \\
A7 & Zaragoza, Coah. & R & A15 & Zaragoza Coah. & R \\
A8 & Cuauhtémoc, Tam. & T & A16 & Cuauhtémoc, Tam. & T \\
\hline
\end{tabular}

Cond. = condición de siembra; $\mathrm{T}$ = temporal (secano); $\mathrm{R}=$ riego; $\mathrm{P}=$ punta de riego.

Cuadro 3. Cuadrados medios del análisis de varianza combinado y regresión de Finlay y Wilkinson (1963), para rendimiento $\left(\mathrm{kg} \mathrm{ha}^{-1}\right)$ de 44 genotipos de sorgo evaluados en 16 ambientes del norte de México.

\begin{tabular}{lrrr}
\hline Fuente de variación & gl & \multicolumn{1}{c}{ SC } & \multicolumn{1}{c}{ CM } \\
\hline Bloques (B) & 3 & 263747835 & $5494747 * *$ \\
Ambientes (A) & 15 & 5184547437 & $345636496 * *$ \\
Genotipos (G) & 43 & 101142758 & $2352157 * *$ \\
Interacción G x A & 645 & 1016773235 & $1576393 * *$ \\
Regresión conjunta & 1 & 2954910 & $2954910 \mathrm{~ns}$ \\
Regresión genotipos & 42 & 106677045 & $2539930 * *$ \\
Regresión ambientes & 14 & 37524704 & $2680336^{* *}$ \\
Residual & 588 & 869616574 & $1478940 * *$ \\
Error & 2064 & 1883206903 & 912406 \\
Total & 2815 & 8449418170 & 3001569 \\
\hline
\end{tabular}

** Significativo a $(\mathrm{P} \leq 0.01)$; gl = grados de libertad; $\mathrm{SC}=$ suma de cuadrados; $\mathrm{CM}=$ cuadrados medios; ns = no significativo. 
El análisis AMMI mostró que los cuatro primeros componentes principales (Cuadro 4) presentan variabilidad de importancia $(\mathrm{P} \leq 0.01)$. Estos cuatro términos multiplicativos explican $28,19,10$ y $9 \%$ de la suma de cuadrados de la interacción, con $57,55,53$ y 51 grados de libertad respectivamente. Aunque Chisi et al. (1996) reportaron en sorgo valores similares a los observados en este estudio $(29,22$ y $19 \%)$, la variabilidad explicada por el ACP1 puede restringir la discriminación de genotipos mediante este método, como lo consignaron Crossa et al. (1990) y Rodríguez et al. (2002). Van Euwijk (1995) señaló que el primer eje representa la variable ambiental hipotética que describe la mayor cantidad de interacción posible y, por tanto, es el que mejor discrimina entre genotipos.

En este estudio el ACP1 superó en casi $10 \%$ la varianza justificada por el ACP2, lo que coincide con Solano et al. (1998). Aunque la magnitud de la variación no fue tan alta en ACP1, ésta fue significativa $(\mathrm{P} \leq 0.01)$, lo que coincide con Michelena et al. (1995). La división de la varianza de la interacción en el modelo AMMI resultó efectiva, dado que el cuadrado medio $(\mathrm{CM})$ para el ACP1 fue seis veces el valor del CM residual; es decir, logró capturar suficiente varianza de la interacción, lo que sugiere que el modelo AMMI explica mejor los valores de la interacción que el modelo de regresión de Finlay y Wilkinson (1963), pues la interacción del modelo AMMI contiene casi $66 \%$ de la suma de cuadrados total (\% SC $\mathrm{ACP} 1+\% \mathrm{SC}$ ambientes + \% SC genotipos). En total, el modelo AMMI retuvo casi $75 \%$ de la suma de cuadrados total (\% SC ambientes $+\%$ SC genotipos + $\%$ SC interacción), mientras que el residual sólo representa $4 \%$.

Los híbridos que presentaron la menor interacción y, por tanto, un comportamiento estable, fueron: 'RB119x435', 'Magnum', 'RB-106x25CEA', 'RB118x430REA', 'RB-119x430CEA', 'Asgrow Coral' y 'WAC-690', con valores de ACP1 de -0.11, 0.41, 0.50, $1.23,-1.49,-1.55 \mathrm{y}-1.84$, respectivamente, que son los más próximos a cero (Zobel et al., 1988). Por el contrario, los genotipos más inestables fueron: 'RB125xBER', 'RB-104xBER', 'RB-119xBER', 'RB-106x216', 'RB-125x430CEA', 'RB-118x430CEA' y 'RB-5x216', con valores de ACP1 de -55.64, -31.10, -22.66, -21.03, $-18.70,18.44$ y 18.09 , respectivamente. Es de destacar que los tres genotipos más inestables tuvieron como progenitor masculino a la línea BER, aunque dos de las cruzas presentaron rendimientos superiores a la media general.

En 2001 los ambientes (Cuadro 6) presentaron una mayor interacción o fueron más inestables, y este año el rendimiento promedio fue mayor que el de 2002. Los ambientes A10 (Empalme, Tam.) y A11 (el Vaso, Tam.) durante 2002 presentaron la menor interacción; en esas localidades los rendimientos fueron inferiores al promedio general. El que la misma localidad presente valores de ACP1 positivos y negativos en diferentes años indica que ahí la variación ambiental es grande, por lo que es conveniente incrementar el número de años de evaluación (González et al., 2007). En la Figura 1 se observa un mayor número de genotipos con valores positivos, algunos con rendimiento superiores al promedio pero con alta interacción G x A ('RB118x25CEA', 'RB104x435', 'RB118x435', 'RB-4040', 'RB116x430', 'Coral, WAC690' y 'RB119x430'), mientras que genotipos como 'DK55', 'RB106x216' y 'RB119xBER' presentaron altos rendimientos y valores de interacción $\mathrm{G}$ x A negativos del ACP1; ambos grupos de genotipos fueron los más inestables.

Se detectó poca o nula asociación entre los híbridos de mayor rendimiento y los ambientes más productivos: A4, A5 y A3 [Abasolo (T), Río Bravo (R) y el Vaso (T)]. Lo mismo sucedió en los ambientes pobres. Además, los ambientes más inestables fueron A15, A13, A12 y A6 (Zaragoza, Coah., Río Bravo, Tam (T), Abasolo, Tam, y Anáhuac, N. L.), con valores positivos; en cambio, A2, A4, A8 y A3 (Empalme, Abasolo, Cuauhtémoc y el Vaso en Tamaulipas durante 2001) presentaron valores negativos.

El modelo AMMI permitió agrupar los ambientes en cuatro grupos relativamente homogéneos, de tal forma que se pueda explotar a los genotipos tanto por su capacidad de adaptación amplia como la específica (Gauch, 1988). El primero grupo incluye a seis ambientes (A1, A9, A10, A11, A12, A14), cinco de los cuales corresponden a 2002; el segundo reúne los ambientes A3, A4, A7 y A16; el tercero, que es menos homogéneo, está formado por A5, A6, A13 y A15; y el cuarto grupo por A2 y A8.

Es decir, el modelo AMMI permitió analizar con detalle la interacción $\mathrm{G}$ x A, esencial en la evaluación del rendimiento de grano de cualquier cultivo, carácter para el cual esa interacción es agronómicamente importante (Crossa et al., 1990; Gauch y Zobel, 1996), lo que hace más eficiente el proceso de selección de genotipos para una región en particular. 


\begin{tabular}{|c|c|c|c|}
\hline Fuentes de variación & gl & $\mathrm{SC}$ & $\mathrm{CM}$ \\
\hline Bloques (B) & 48 & 263747835 & $5494747 * *$ \\
\hline Ambientes (A) & 15 & 5184547437 & $345636496 * *$ \\
\hline Genotipos (G) & 43 & 101142758 & $2352157 * *$ \\
\hline Interacción $\mathrm{G}$ x A & 645 & 1016773235 & $1576393 * *$ \\
\hline $\mathrm{ACP} 1$ & 57 & 286799425 & $5031569 * *$ \\
\hline $\mathrm{ACP} 2$ & 55 & 193371113 & $3515838 * *$ \\
\hline ACP3 & 53 & 105820440 & $1996612 * *$ \\
\hline $\mathrm{ACP} 4$ & 51 & 86454126 & $1695179 * *$ \\
\hline Residual & 429 & 344328131 & $802630 \mathrm{~ns}$ \\
\hline Error & 2064 & 1883206904 & 912406 \\
\hline Total & 2815 & 8449418170 & 3001569 \\
\hline
\end{tabular}

Cuadro 5. Rendimiento medio y valores de ACP1 para 44 genotipos de sorgo en 16 ambientes del noreste de México, durante los ciclos otoñoinvierno 2001 y 2002.

\begin{tabular}{|c|c|c|c|c|c|c|c|}
\hline Núm. & Híbrido & $\begin{array}{l}\text { Rend. } \\
\left(\mathrm{kg} \mathrm{ha}^{-1}\right)\end{array}$ & ACP1 & Núm. & Híbrido & $\begin{array}{l}\text { Rendto. } \\
\left(\mathrm{kg} \mathrm{ha}^{-1}\right)\end{array}$ & $\mathrm{ACP} 1$ \\
\hline G1 & 'RB-118x430' & 3200 & 6.54 & G23 & 'RB-119X435' & 3277 & -0.11 \\
\hline $\mathrm{G} 2$ & 'RB-118x435' & 3416 & 5.36 & G24 & 'RB-119X216' & 2939 & -8.43 \\
\hline G3 & 'RB-118x204' & 3019 & 11.34 & G25 & 'RB-119XBER' & 3258 & -22.66 \\
\hline G4 & 'RB-118x216' & 3190 & 9.60 & G26 & 'RB-119X430CEA' & 2935 & -1.49 \\
\hline G5 & 'RB-118x430REA' & 3197 & 1.23 & G27 & 'RB-119X25CEA' & 3087 & 8.28 \\
\hline G6 & 'RB-118x430CEA' & 3225 & 18.44 & G28 & 'RB-116X430' & 3376 & 14.53 \\
\hline G7 & 'RB-118x25CEA' & 3577 & 15.86 & G29 & 'RB-116X435' & 3203 & 14.31 \\
\hline G8 & 'RB-Patrón' & 3173 & -5.11 & G30 & 'RB-116X216' & 3021 & 11.69 \\
\hline G9 & 'RB-104x435' & 3437 & 12.43 & G31 & 'RB-116X25CEA' & 3134 & 5.80 \\
\hline G10 & 'RB-104x216' & 3199 & 2.57 & G32 & 'RB-106X430' & 3099 & -7.43 \\
\hline G11 & 'RB-104x430CEA' & 3112 & 3.01 & G33 & 'RB-106X435' & 3049 & 3.38 \\
\hline G12 & 'RB-104xBER' & 3215 & -31.10 & G34 & 'RB-106X204' & 3201 & 5.34 \\
\hline G13 & 'RB-5x435’ & 2998 & 9.88 & G35 & 'RB-106X216' & 3263 & -21.03 \\
\hline G14 & 'RB-5x204' & 3125 & -5.20 & G36 & 'RB-106X25CEA' & 3103 & 0.50 \\
\hline G15 & 'RB-5x216' & 3108 & 18.09 & G37 & 'Asgrow Coral' & 3372 & -1.55 \\
\hline G16 & 'RB-5x430REA' & 3172 & 2.95 & G38 & 'RB-4040' & 3404 & -2.60 \\
\hline G17 & 'RB-5x430CEA' & 3191 & 2.48 & G39 & 'RB-3030' & 2938 & 11.58 \\
\hline G18 & 'RB-5x25CEA' & 3080 & 6.13 & G40 & 'RB-3006' & 3188 & 3.60 \\
\hline G19 & 'RB-125x430CEA' & 3101 & -18.70 & G41 & 'Magnum 380' & 3266 & 0.41 \\
\hline G20 & 'RB-125x25CEA' & 2866 & -14.22 & G42 & 'Pioneer 8313' & 2713 & -3.74 \\
\hline G21 & 'RB-125xBER' & 2791 & -55.64 & G43 & 'Dekalb D55' & 3715 & -14.52 \\
\hline $\mathrm{G} 22$ & 'RB-119x430' & 3336 & 9.99 & G44 & 'WAC 690' & 3359 & -1.84 \\
\hline
\end{tabular}

Rend. = rendimiento; ACP1 $=$ componente principal 1.

Cuadro 6. Valores de ACP1 para 16 ambientes del noreste de México, durante los ciclos otoño-invierno 2001 y 2002.

\begin{tabular}{|c|c|c|c|c|c|c|c|}
\hline Núm. & Localidades 2001 & $\begin{array}{l}\text { Rend. } \\
\left(\mathrm{kg} \mathrm{ha}^{-1}\right)\end{array}$ & ACP1 & Núm. & Localidades 2002 & $\begin{array}{l}\text { Rend. } \\
\left(\mathrm{kg} \mathrm{ha}^{-1}\right)\end{array}$ & ACP1 \\
\hline$\overline{\mathrm{A} 1}$ & Río Bravo, Tam. (T) & 2571 & 13.71 & A9 & Río Bravo, Tam. (T) & 2195 & 13.49 \\
\hline A2 & Empalme, Tam. (T) & 2853 & -53.64 & A10 & Empalme, Tam. (T) & 2280 & 0.19 \\
\hline $\mathrm{A} 3$ & El Vaso, Tam. (T) & 4557 & -20.06 & A11 & El Vaso, Tam. (T) & 1572 & 4.44 \\
\hline A4 & Abasolo, Tam. (R) & 5796 & -25.32 & $\mathrm{~A} 12$ & Abasolo, Tam. (P) & 1521 & 21.12 \\
\hline A5 & Río Bravo, Tam. (R) & 5546 & 5.02 & A13 & Río Bravo, Tam. (R) & 4520 & 24.79 \\
\hline A6 & Anahuac, N. L. (R) & 3570 & 21.56 & A14 & González V, Tam. (T) & 1347 & 7.12 \\
\hline A7 & Zaragoza, Coah. (R) & 3518 & -12.39 & A15 & Zaragoza Coah. (R) & 3122 & 39.99 \\
\hline \multirow[t]{2}{*}{ A8 } & Cuauhtémoc, Tam.(T) & 1915 & -21.98 & A16 & Cuauhtémoc, Tam. (T) & 3888 & -17.93 \\
\hline & Media & 3790 & & & Media & 2555 & \\
\hline
\end{tabular}

Rend. = rendimiento; $\mathrm{T}$ = temporal (secano); $\mathrm{R}=$ riego; $\mathrm{P}=$ punta de riego; $\mathrm{ACP} 1$ = componente principal 1. 


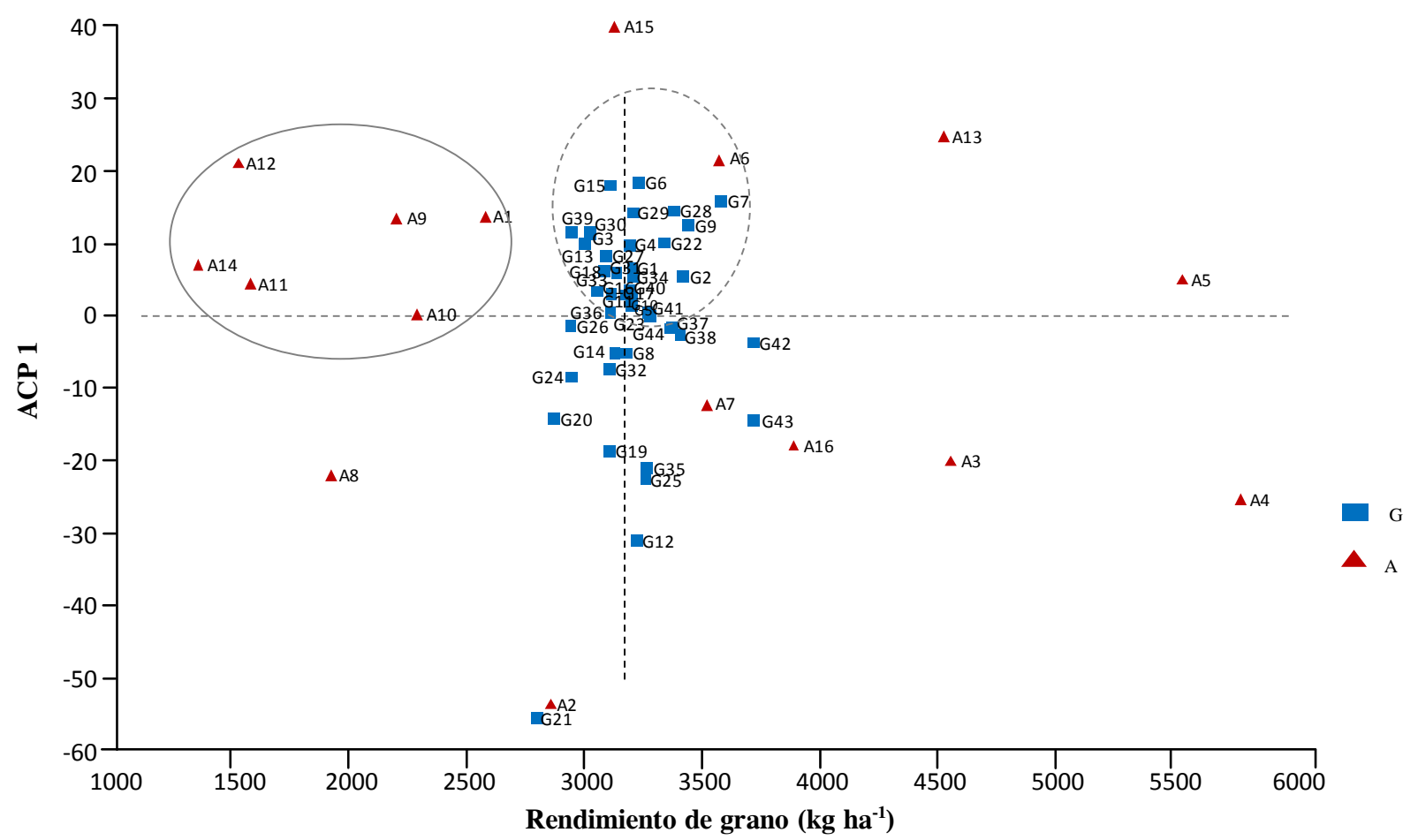

Figura 1. Biplot de coordenadas del primer eje del análisis de componentes principales (ACP1) en función del rendimiento de grano, para 44 genotipos (G) de sorgo y 16 ambientes (A) del noreste de México.

\section{CONCLUSIONES}

Existen diferencias en el comportamiento entre híbridos de sorgo en los diversos ambientes evaluados. El modelo AMMI, además de ser informativo y sencillo de interpretar, resultó más efectivo para caracterizar la respuesta de los genotipos que el análisis de regresión de Finlay y Wilkinson. Los genotipos de sorgo más estables fueron 'RB-119x435', 'Magnum', 'RB-106x25CEA', 'RB118x430REA', 'RB-119x430CEA', 'Asgrow Coral' y 'WAC-690'. No se observó asociación entre los híbridos más productivos y los ambientes de mayor producción.

\section{AGRADECIMIENTOS}

Trabajo financiado por la Fundación Produce Tamaulipas, A. C. a través del proyecto No. 2016247A, "Obtención de híbridos y/o variedades de sorgo para grano con tolerancia a sequía y enfermedades para el noreste de México".

\section{BIBLIOGRAFÍA}

Brancourt-Hulmel M, C Lecomte (2003) Effect of environmental variates on genotype environment interaction of winter wheat: A comparison of biadditive factorial regression to AMMI. Crop Sci. 43:608-617.
Brennan P S, D E Byth, D W Draker, I H De Lacy, D G Butler (1981) Determination of the location and number of test environments for a wheat cultivar evaluation program. Austr. J. Res. 32:189-201.

Chisi M, P Bramel-Cox, M Witt, M Classen, D Andrews (1996) Breeding for grain yield stability using full-sib family recurrent selection in sorghum. Crop Sci. 36:1083-1085.

Crossa J, H G Gauch Jr, R W Zobel (1990) Additive main effects and multiplicative interaction analysis of two international maize cultivar trails. Crop Sci. 30:493-500.

Crossa J, P N Fox, W H Pfeiffer, S Rajaram, H G Gauch Jr (1991) AMMI adjustment for statistical analysis of two international maize cultivar trials. Theor. Appl. Genet. 81:27-37.

Eberhart S A, W A Russell (1966) Stability parameters for comparing varieties. Crop Sci. 6:36-46.

Finlay K W, A A Wilkinson (1963) The analysis of adaptation in a plant breeding program. Austr. J. Agric. Res. 14:742-754.

Gauch H G Jr (1988) Model selection and validation for yield trials with interaction. Biometrics 44:705-715.

Gauch H G Jr (1992) Statistical analysis of regional yield trials: AMMI analysis of factorial designs. Elsevier, New York, New York. $278 \mathrm{p}$.

Gauch H G Jr, Furnas R E (1991) Statistical analysis of yield trails with MATMODEL. Agron. J. 83:916-920.

Gauch H G Jr, R Zobel (1996) AMMI analysis of yield trials. In: Genotype-by-Environment Interaction M S Kang and H G Gauch Jr (eds). CRC Press. Boca Ratón. pp:85-122.

Gollob H F (1968) A statistical model which combines features of factor analysis of variance techniques. Psycometrika 33:73-115.

González T, E Monteverde, C Marín, I P M Madriz (2007) Comparación de tres métodos para estimar estabilidad del rendimiento en nueve variedades de algodón. Interciencia 32:344-348. 
Hernández M, J Crossa (2000) El análisis AMMI y la gráfica Biplot en SAS. Disponible en: www.cimmyt.cgiar.org/biometrics (Mayo 2008).

Lin C S, M R Binns, L P Lefkovitch (1986) Stability analysis: Where do we stand? Crop Sci. 26:894-900.

Mandel J (1971) A new analysis of variance model for non-additive data. Technometrics 13:1-18.

Michelena A, I Romagosa, J Martin, A López (1995) Influencia ambiental y varietal en diferentes parámetros de calidad y rendimiento en trigo duro. Investigación Agraria: Prod. Protec. Veg. 10:191-200.

Montes G N, J Aguirre R (1992) Sorgo. In: Manual de Cultivos del Norte de Tamaulipas, Patronato para la Investigación Fomento y Sanidad Vegetal. SARH. Matamoros, Tamaulipas, México. pp:54-63.

Rodríguez P J E, J Sahagún C, H E Villaseñor M, J D Molina G, A Martínez G (2002) Estabilidad de siete variedades comerciales de trigo (Triticum aestivum L.) de temporal. Rev. Fitotec. Mex. 25:143-151.

Shukla G K (1972) Some statistical aspects of partitioning genotypeenvironmental components of variability. Heredity 29:237-245.
Solano S J, P Barriga B, A Krarup H, H Figueroa S (1998) Estabilidad temporal del rendimiento de genotipos mutantes de trigo, mediante el modelo de interacción multiplicativa y efectos principales aditivos (AMMI: Additive Main effect and Multiplicative Interaction model). Agro Sur 26:19-32.

Van Eeuwijk F (1995) Linear and bilinear models for the analysis of multi-environment trials: I. An inventory of models. Euphytica 84:1-7.

Vargas H M, J Crossa (2000) El análisis AMMI y la gráfica del biplot en SAS. Unidad de biometría. CIMMYT. México. Disponible en: www.cimmyt.cgiar.org/biometrics (Mayo 2008).

Yan W, L A Hunt, Q Sheng, Z Szlavnics (2000) Cultivar evaluation and mega-environment investigation based on the GGE biplot. Crop Sci. 40:597-605.

Yates F, W G Cochran (1938) The analysis of groups of experiments. J. Agric. Sci. (Cambridge) 28:556-580.

Zobel $R$ (1990) A powerful statistical model for understanding genotype-by-environment interaction. In: Genotype-byEnvironment Interaction and Plant Breeding. M S Kang (ed) Louisiana State University. pp:126-140.

Zobel R, M Wright, H Gauch (1988) Statistical analysis of a yield trial. Agron. J. 80:88-397. 